\title{
Phylogenetic placement of the enigmatic parasite, Polypodium hydriforme, within the Phylum Cnidaria Nathaniel M Evans ${ }^{1}$, Alberto Lindner ${ }^{2}$, Ekaterina V Raikova ${ }^{3}$, Allen G Collins ${ }^{4}$ and Paulyn Cartwright*1
}

Address: ${ }^{1}$ Department of Ecology and Evolutionary Biology, University of Kansas, Lawrence, Kansas 66045, USA, ${ }^{2}$ CEBIMar, University of São Paulo, São Sebastião, Brazil, ${ }^{3}$ Institute of Cytology of the Russian Academy of Sciences, St. Petersburg, Russia and ${ }^{4}$ National Systematics Laboratory of NOAA Fisheries Service, National Museum of Natural History, Smithsonian Institution, Washington, DC 20013-7012, USA

Email: Nathaniel M Evans - evansnat@ku.edu; Alberto Lindner - alindner@usp.br; Ekaterina V Raikova - raikova@swipnet.se; Allen G Collins - COLLINSA@si.edu; Paulyn Cartwright* - pcart@ku.edu

* Corresponding author

\section{Published: 9 May 2008}

BMC Evolutionary Biology 2008, 8:139 doi:10.1 |86//47|-2/48-8-139
Received: 4 December 2007

Accepted: 9 May 2008

This article is available from: http://www.biomedcentral.com/I47I-2/48/8/139

(c) 2008 Evans et al; licensee BioMed Central Ltd.

This is an Open Access article distributed under the terms of the Creative Commons Attribution License (http://creativecommons.org/licenses/by/2.0), which permits unrestricted use, distribution, and reproduction in any medium, provided the original work is properly cited.

\begin{abstract}
Background: Polypodium hydriforme is a parasite with an unusual life cycle and peculiar morphology, both of which have made its systematic position uncertain. Polypodium has traditionally been considered a cnidarian because it possesses nematocysts, the stinging structures characteristic of this phylum. However, recent molecular phylogenetic studies using $18 \mathrm{~S}$ rDNA sequence data have challenged this interpretation, and have shown that Polypodium is a close relative to myxozoans and together they share a closer affinity to bilaterians than cnidarians. Due to the variable rates of I8S rDNA sequences, these results have been suggested to be an artifact of long-branch attraction (LBA). A recent study, using multiple protein coding markers, shows that the myxozoan Buddenbrockia, is nested within cnidarians. Polypodium was not included in this study. To further investigate the phylogenetic placement of Polypodium, we have performed phylogenetic analyses of metazoans with $18 \mathrm{~S}$ and partial $28 \mathrm{~S}$ rDNA sequences in a large dataset that includes Polypodium and a comprehensive sampling of cnidarian taxa.
\end{abstract}

Results: Analyses of a combined dataset of $18 \mathrm{~S}$ and partial $28 \mathrm{~S}$ sequences, and partial $28 \mathrm{~S}$ alone, support the placement of Polypodium within Cnidaria. Removal of the long-branched myxozoans from the I8S dataset also results in Polypodium being nested within Cnidaria. These results suggest that previous reports showing that Polypodium and Myxozoa form a sister group to Bilateria were an artifact of long-branch attraction.

Conclusion: By including 28S rDNA sequences and a comprehensive sampling of cnidarian taxa, we demonstrate that previously conflicting hypotheses concerning the phylogenetic placement of Polypodium can be reconciled. Specifically, the data presented provide evidence that Polypodium is indeed a cnidarian and is either the sister taxon to Hydrozoa, or part of the hydrozoan clade, Leptothecata. The former hypothesis is consistent with the traditional view that Polypodium should be placed in its own cnidarian class, Polypodiozoa. 


\section{Background}

Polypodium hydriforme is an endocellular parasite whose unusual life cycle, peculiar morphology, and high rates of DNA evolution, have led to much controversy regarding its phylogenetic position within metazoans [1-5]. Polypodium spends most of its life inside the oocytes of acipenseriform fishes (sturgeons and paddlefish). During this time, Polypodium develops from a binucleate cell into an inside-out planuliform larva and then into an elongate inside-out stolon; the epidermal cell layer is located internal to the body and the gastrodermis is located externally [6-8]. The embryo, larva and stolon are surrounded by a protective polyploid cell, which also functions in digestion [7]. Just prior to host spawning, Polypodium everts to the normal position of cell layers, revealing tentacles scattered along the stolon. During eversion, the yolk of the host oocyte fills the gastral cavities of the parasite, supplying the future free-living stage with nutrients $[6,7]$. Finally, upon emerging from the host egg in fresh water, the freeliving stolon (Figure 1A) fragments into individual medusoid-like forms (Figure 1B) that go on to multiply by means of longitudinal fission, form sexual organs, and ultimately infect host fish with their gametophores [6-9].

Two conflicting hypotheses have been proposed regarding the phylogenetic placement of Polypodium. The first, more traditional hypothesis is that Polypodium is a cnidarian. Some have suggested it is nested within a derived group of hydrozoans, the Narcomedusae [10-13] or the cnidarian class Scyphozoa [14]; while others have suggested it belongs to a separate cnidarian class, Polypodiozoa $[1,15,16]$. The assignment of Polypodium to Cnidaria is based primarily on morphological evidence, most notably the fact that Polypodium possesses nematocysts $[17,18]$, the stinging structures characteristic of all cnidarians. In addition, the presence of tentacles and overall body-plan organization of Polypodium are reminiscent of cnidarians, although it is unclear if the adult free-living stage is homologous to a polyp or medusa stage. This hypothesis is supported by a cladistic analysis of small subunit nuclear ribosomal DNA (18S rDNA) sequences in conjunction with morphological characters (including nematocysts) [2]. In this study, Polypodium falls within the medusozoan clade of cnidarians, although the non-cnidarian placozoan, Trichoplax $[19,20]$, also fell within this clade, rendering Cnidaria paraphyletic.

The second hypothesis is that Polypodium is the sister taxon to Myxozoa, a diverse group of parasites in aquatic animals, and that Polypodium + Myxozoa is the sister group to Bilateria [2-4]. This hypothesis is derived from cladistic analyses utilizing 18S rDNA sequences [2-4]. However,
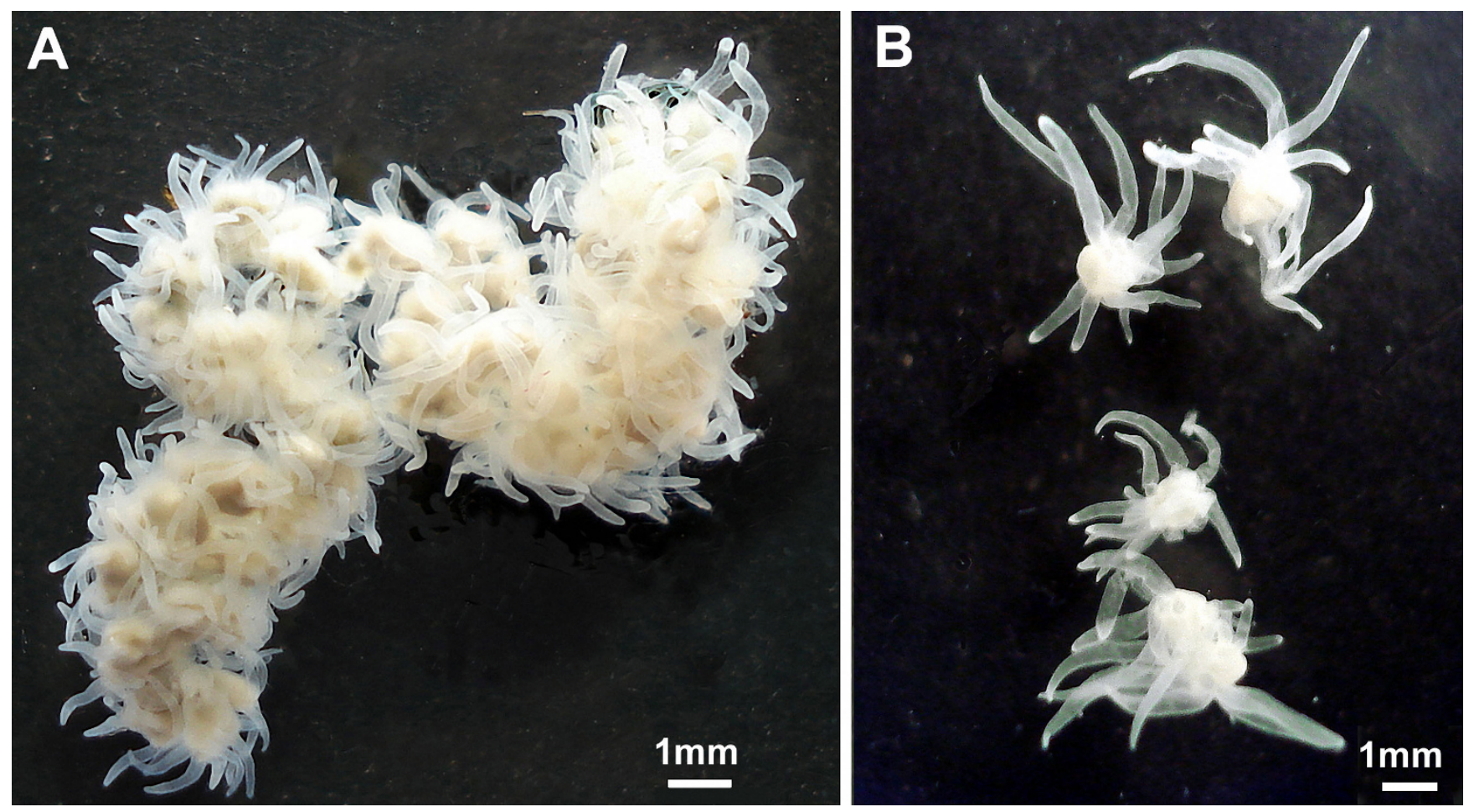

Figure I

Polypodium hydriforme. A) Stolon stage just after emerging from the host oocyte. B) Four specimens of free-living Polypodium with 12 tentacles. Photos by E. Raikova. 
because Polypodium and myxozoans have unusually high divergence rates in their $18 \mathrm{~S}$ rDNA sequences, these cladistic analyses have been criticized by a number of authors who suggest that the data might be unduly affected by long-branch attraction (LBA) [5,21,22]. Despite some attempts to overcome the effects of LBA through the use of a maximum likelihood (ML) approach [21-23] and pruning long branches [5,22], these results have been largely silent on the placement of Polypodium. For instance, Kim et al. [22] applied a maximum likelihood approach to $18 \mathrm{~S}$ rDNA sequence data and found that myxozoans and Polypodium did not group together. Instead, Polypodium was part of an unresolved polytomy that included several cnidarian lineages and Trichoplax, as well as myxozoans + Bilateria. Most recently, JimenezGuri et al. [24] utilized multiple protein-coding gene sequences in a ML analysis and found the myxozoan, Buddenbrockia plumatellae nested within cnidarians. Unfortunately, this study had relatively limited sampling of cnidarians and did not include Polypodium.

In an attempt to resolve this controversy, we sequenced an additional marker in Polypodium, a partial gene sequence of the large nuclear ribosomal unit (28S rDNA), and greatly expanded the taxonomic sampling of cnidarian sequences. Using this approach, we provide evidence that Polypodium is nested within Cnidaria and does not group with myxozoans.

\section{Results}

\section{Sampled taxa}

All taxa used in this study are arranged taxonomically in Table 1. 155 sequences were obtained from GenBank. 45 new cnidarian sequences for $18 \mathrm{~S}$ and 59 for $28 \mathrm{~S}$ (including 2 new 18S and 2 new partial 28S from Polypodium taxa) were generated for this study and deposited in GenBank (see Table 1 for accession numbers). Polypodium hydriforme sequences were obtained from both North American and Eurasian hosts. Eurasian samples were collected from two individuals of Acipenser ruthenus. North American samples were collected from Polyodon spathula and Scaphirhynchus platorynchus. This is the first reported presence of Polypodium infection in Scaphirhynchinae. While Polypodium was recovered from the oocytes of $S$. platorynchus, the sample from which we extracted sequence data was found externally attached to its presumed host. More specific collection data for Polypodium specimens are associated with each sequence submitted to GenBank (see Table 1 for accession numbers).

All Polypodium sequences were newly generated for this study. We did not include the previously published $18 \mathrm{~S}$ Polypodium sequence (GenBank accession number U37526) because of concern over the quality of the sequence which included a number of ambiguities. Fur- thermore, while the two new Polypodium $18 \mathrm{~S}$ sequences (from hosts Acipenser ruthensus and Polyodon spathula) differed from each other by a total of 8 sites they differed from \#U37526 by 77 and 83 sites respectively. These differences included a large number of insertions and deletions. The two new $28 \mathrm{~S}$ sequences (from hosts Acipenser ruthensus and Scaphirhynchus platorynchus) only differed from each other by 2 sites.

\section{Position of Polypodium}

The complete combined dataset of $18 \mathrm{~S}$ rDNA and partial 28 rDNA contains 4842 characters, 2901 of which are variable and 2124 parsimony informative. Both the $\mathrm{ML}$ and parsimony topologies reconstructed from the combined dataset suggest that Polypodium is nested within a monophyletic Cnidaria, and myxozoans are the sister taxon to bilaterians (Figure 2). The ML bootstrap values supporting a monophyletic Cnidaria (including Polypodium), a monophyletic Medusozoa (including Polypodium) and the Polypodium + hydrozoan clade are 73, 67 and 73 respectively (Figure $2 \mathrm{~A}$, and Additional file 1 ). Parsimony analysis of the combined dataset differs from that of ML in that Polypodium is nested within a group of hydrozoans, the leptothecates (Figure 2B). The parsimony bootstrap values supporting a monophyletic Cnidaria and Hydrozoa, with Polypodium nested within these clades are 50 and 51 respectively (Figure $2 \mathrm{~B}$ ). The clade nested within hydrozoans, that includes Polypodium + leptothecates is weakly supported in the sub-sampling tests with a bootstrap value of less than 50 .

The analyses using partial 28S rDNA sequences alone (129 sampled taxa) contains 1756 characters, 1196 of which are parsimony informative. The ML topology using this dataset reveals Polypodium nested within Cnidaria, specifically within leptothecate hydrozoans, (Additional file 2). This analysis however fails to recover a monophyletic Cnidaria, as the anthozoans are placed outside the Cnidaria + Bilateria clade. Analysis of the 18S rDNA dataset alone (132 taxa, 3038 characters, 1469 parsimony informative) under both optimality criteria conflicts with the combined and partial $28 \mathrm{~S}$ topologies. The $18 \mathrm{~S}$ rDNA topology for both criteria place Polypodium at the base of Bilateria (Figure 3A, Additional files 3, 4 and 5). However, the ML topology also reflects a sister relationship between Polypodium and myxozoans (Figure $3 \mathrm{~A}$ and Additional file 3A) while the parsimony topology does not (Additional files 4 and 5). Moreover, under parsimony criteria the position of myxozoans is dependent upon how gaps are coded: if gaps are coded as a fifth character state, myxozoans are placed as a highly derived clade of bilaterians (Additional file 4); if gaps are coded as missing, myxozoans are placed as sister to all metazoans (Additional file 5 ). The $18 \mathrm{~S}$ analysis showing placement of Polypodium with Bilateria, and more specifically as sister to myxo- 
Table I: Taxon and sequence list

\begin{tabular}{|c|c|c|c|c|}
\hline \multirow[b]{2}{*}{ Higher classification } & \multirow[b]{2}{*}{ Taxon ID } & \multicolumn{2}{|c|}{ Accession numbers } & \multirow[b]{2}{*}{ Voucher } \\
\hline & & $28 S$ & $18 S$ & \\
\hline \multicolumn{5}{|l|}{ Bilateria } \\
\hline Annelida & Proceraea cornuta & $\underline{A F 212165}$ & $\underline{A F 212179}$ & \\
\hline Annelida & Urechis caupo & $\overline{\mathrm{AF} 342804}$ & $\overline{\mathrm{AF} 342805}$ & \\
\hline Arthropoda & Limulus polyphemus & $\mathrm{AF} 212167$ & $\underline{\text { U9|490 }}$ & \\
\hline Arthropoda & Tenebrio sp./Tenebrio molitor & $\overline{\mathrm{AY} 210843}$ & $\times 07801$ & \\
\hline Brachiopoda & Phoronis vancouverensis & AF342797 & $\underline{\mathrm{U} 2648}$ & \\
\hline Chordata & Oncorhynchus sp./O. kisutch & $\underline{\mathrm{U} 3434 \mathrm{I}}$ & AF030250 & \\
\hline Chordata & Petromyzon marinus & AF061798 & $\overline{M 97575.1}$ & \\
\hline Chordata & Raja schmidti & AF278683 & AF278682 & \\
\hline Chordata & Triakis semifasciata & $\mathrm{AF} 212182$ & AF212180 & \\
\hline Echinodermata & Strongylocentrotus purpuratus & $\mathrm{AF} 212171$ & $\mathrm{~L} 28056$. & \\
\hline Hemichordata & Cephalodiscus gracilis & $\overline{\mathrm{AF} 212172}$ & AF236798 & \\
\hline Hemichordata & Harrimania sp. & $\overline{\mathrm{AF} 212173}$ & $\overline{\mathrm{AF} 236799}$ & \\
\hline Hemichordata & Ptychodera flava & $\mathrm{AF} 212176$ & AF27868I & \\
\hline Hemichordata & Ptychoderidae & $\overline{\mathrm{AF} 278684}$ & D14359 & \\
\hline Hemichordata & Saccoglossus kowalevskii & $\overline{\mathrm{AF} 212175}$ & $\overline{\mathrm{L} 28054}$ & \\
\hline Kinorhyncha & Pycnophyes sp.Tjarno & AY859597 & AY859598 & \\
\hline Mollusca & Parvicardium minimum & $\overline{\mathrm{DQ} 279966}$ & $\overline{\mathrm{DQ} 279942}$ & \\
\hline Mollusca & Placopecten magellanicus & $\mathrm{AF} 342798$ & $\times 53899$ & \\
\hline Nematoda & Caenorhabditis elegans & $\underline{X 03680}$ & $\underline{X 03680}$ & \\
\hline Nematomorpha & Chordodes morgani & $\mathrm{AF} 342787$ & AF036639 & \\
\hline Nemertea & Amphiporus sp. & $\mathrm{AF} 342786$ & $\mathrm{AFI} 19077$ & \\
\hline Nemertodermatida & Meara stichopi & $\overline{\mathrm{AY} 157605}$ & $\overline{\mathrm{AFI} I 9085}$ & \\
\hline Onychophora & Peripatus sp. & AY210836 & AY210837 & \\
\hline Platyhelminthes & Diclidophora denticulata & AYI57169 & A]228779 & \\
\hline Platyhelminthes & Stenostomum leucops & $\overline{A Y 157151}$ & $\mathrm{D} 85095$ & \\
\hline Platyhelminthes & Stylochus zebra & $\overline{\mathrm{AF} 342800}$ & $\mathrm{AF} 342801$ & \\
\hline Priapulida & Priapulus caudatus & AY210840 & Z38009 & \\
\hline Sipuncula & Phascolopsis gouldii & AF342795 & $\mathrm{AF342796}$ & \\
\hline Tardigrada & Milnesium.sp.M. tardigradum & AY210826 & U49909 & \\
\hline Urochordata & Styela plicata & $\overline{\mathrm{AFI} 58724}$ & $\underline{\underline{L} 12444}$ & \\
\hline Urochordata & Thalia democratica & AFI58725 & D|4366 & \\
\hline \multicolumn{5}{|l|}{ Cnidaria } \\
\hline Polypodiozoa & Polypodium (Host: Acipenser ruthenus) & EU272585 & EU272630 & \\
\hline Polypodiozoa & Polypodium (Host: Polyodon spathula) & & $\overline{\text { EU272629 }}$ & \\
\hline Polypodiozoa & Polypodium (Host:Scaphirhynchus platorynchus) & EU272586 & & \\
\hline Anthozoa, Antipatharia & Antipathes galapagensis & $\overline{\mathrm{AY} 026365}$ & $\underline{\mathrm{AF} I 00943}$ & \\
\hline Anthozoa, Scleractinia & Montastraea franksi & $\overline{\mathrm{AY} 026375}$ & $\overline{\text { AY026382 }}$ & \\
\hline Cubozoa, Carybdeidae & Carybdea rastonii & AY920787 & $\mathrm{AF} 358108$ & \\
\hline Cubozoa, Carybdeidae & Darwin carybdeid sp. & $\overline{\mathrm{AY} 920788}$ & $\overline{\mathrm{AF} 358105}$ & \\
\hline Cubozoa, Carybdeidae & Tripedalia cystophora & EU272595 & EU272637 & \\
\hline Cubozoa, Chirodropidae & Chironex fleckeri & AY920785 & AF358104 & \\
\hline Cubozoa, Chirodropidae & Chiropsalmus sp. & AY920786 & $\mathrm{AF358103}$ & \\
\hline Hydrozoa, Capitata & Dipurena ophiogaster & EU272560 & EU272615 & KUNHM 2803 \\
\hline Hydrozoa, Capitata & Ectopleura dumortieri & EU272561 & EU272616 & \\
\hline Hydrozoa, Capitata & Euphysora bigelowi & EU272563 & EU272618 & KUNHM 2829 \\
\hline Hydrozoa, Capitata & Moerisia sp. & AY920801 & $\mathrm{AF358083}$ & \\
\hline Hydrozoa, Capitata & Pennaria disticha & $\overline{\text { EU272581 }}$ & AY920762 & \\
\hline Hydrozoa, Capitata & Polyorchis penicillatus & & $\mathrm{AF} 358090$ & \\
\hline Hydrozoa, Capitata & Porpita sp. & AY920803 & AF358086 & \\
\hline Hydrozoa, Capitata & Ralpharia gorgoniae & EU272590 & EU272633 & KUNHM 2778 \\
\hline Hydrozoa, Capitata & Scrippsia pacifica & AY920804 & $\mathrm{AF358091}$ & \\
\hline Hydrozoa, Capitata & Solanderia ericopsis & $\overline{\text { EU272593 }}$ & EU272636 & MHNG INVE29593 \\
\hline Hydrozoa, Capitata & Velella sp. & $\overline{\text { EU272597 }}$ & $\overline{\mathrm{AF} 358087}$ & \\
\hline Hydrozoa, Capitata & Zanclea prolifera & EU272598 & EU272639 & KUNHM 2793 \\
\hline Hydrozoa, Capitata & Zyzzyzus warreni & EU272599 & EU272640 & KUNHM 2777 \\
\hline Hydrozoa, Capitata & Candelabrum cocksii & AY920796 & AY920758 & MHNG INVE2953I \\
\hline
\end{tabular}


Table I: Taxon and sequence list (Continued)

\begin{tabular}{|c|c|c|c|c|}
\hline Hydrozoa, Capitata & Cladocoryne floccosa & EU27255I & EU272608 & \\
\hline Hydrozoa, Filifera & Bimeria vestita & EU272548 & EU272605 & \\
\hline Hydrozoa, Filifera & Bougainvillia carolinensis & $\underline{E U 272549}$ & EU272606 & \\
\hline Hydrozoa, Filifera & Brinckmannia hexactinellidophila & EU272550 & EU272607 & MHNG INVE38I48 \\
\hline Hydrozoa, Filifera & Clava multicornis & EU272552 & EU272609 & \\
\hline Hydrozoa, Filifera & Clavactinia gallensis & $\underline{\text { EU272553 }}$ & EU272610 & MHNG INVE33470 \\
\hline Hydrozoa, Filifera & Cordylophora caspia & EU272556 & $\overline{\text { EU272612 }}$ & \\
\hline Hydrozoa, Filifera & Corydendrium sp. & EU272557 & $\underline{E U 272613}$ & KUNHM 2764 \\
\hline Hydrozoa, Filifera & Dicoryne conybearei & EU272559 & EU272614 & MHNG INVE32949 \\
\hline Hydrozoa, Filifera & Eudendrium.racemosum & EU272562 & EU272617 & \\
\hline Hydrozoa, Filifera & Fabienna sphaerica & AY920797 & AY920767 & \\
\hline Hydrozoa, Filifera & Garveia annulata/Garveia sp. & EU272564 & AY920766 & KUNHM 2860 \\
\hline Hydrozoa, Filifera & Hydra circumcincta & AY026371 & AF358080 & \\
\hline Hydrozoa, Filifera & Hydractinia symbiolongicarpus & EU272568 & EU272621 & \\
\hline Hydrozoa, Filifera & Hydrichthella epigorgia & EU272569 & EU272622 & KUNHM 2665 \\
\hline Hydrozoa, Filifera & Hydrichthys boycei & EU272570 & & MHNG INVE374I 7 \\
\hline Hydrozoa, Filifera & Koellikerina fasciculata & EU272571 & EU272623 & \\
\hline Hydrozoa, Filifera & Leuckartiara octona & EU272573 & EU272624 & \\
\hline Hydrozoa, Filifera & Lizzia blondina & $\overline{\text { EU272574 }}$ & $\overline{\text { EU272625 }}$ & \\
\hline Hydrozoa, Filifera & Pachycordyle pusilla & EU272579 & EU272627 & MHNG INVE32953 \\
\hline Hydrozoa, Filifera & Pandea sp. & EU272580 & AY920765 & \\
\hline Hydrozoa, Filifera & Podocoryne carnea & AY920802 & AF358092 & \\
\hline Hydrozoa, Filifera & Proboscidactyla ornata & EU272587 & EU272631 & KUNHM 2767 \\
\hline Hydrozoa, Filifera & Pruvotella grisea & EU272588 & EU272632 & MHNG INVE34436 \\
\hline Hydrozoa, Filifera & Rathkea octopunctata & EU272591 & EU272634 & KUMIP 3I432I \\
\hline Hydrozoa, Filifera & Rhizogeton nudus & $\overline{\text { EU272592 }}$ & $\overline{\text { EU272635 }}$ & MHNG INVE35757 \\
\hline Hydrozoa, Filifera & Turritopsis dohrnii & EU272596 & EU272638 & MHNG INVE29753 \\
\hline Hydrozoa, Leptothecata & Abietinaria filicula & EU272540 & EU272600 & MHNG INVE29947 \\
\hline Hydrozoa, Leptothecata & Aglaophenia tubiformis & $\overline{\text { EU272543 }}$ & $\overline{\text { EU272601 }}$ & MHNG INVE29967 \\
\hline Hydrozoa, Leptothecata & Amphisbetia minima & EU272544 & EU272602 & MHNG INVE2507I \\
\hline Hydrozoa, Leptothecata & Anthohebella parasitica & EU272545 & EU272603 & MHNG INVE29762 \\
\hline Hydrozoa, Leptothecata & Clytia noliformis & EU272554 & EU27261I & \\
\hline Hydrozoa, Leptothecata & Halecium muricatum & $\underline{\text { EU272565 }}$ & EU272619 & MHNG INVE29028 \\
\hline Hydrozoa, Leptothecata & Halopteris minuta & $\overline{\text { EU272567 }}$ & $\overline{\text { EU272620 }}$ & MHNG INVE25073 \\
\hline Hydrozoa, Leptothecata & Melicertum octocostatum & $\underline{E U 272575}$ & AY920757 & USNM 1073342 \\
\hline Hydrozoa, Leptothecata & Octophialucium indicum & EU272577 & EU272626 & MHNG INVE29970 \\
\hline Hydrozoa, Leptothecata & Plumularia setacea & EU272583 & $\overline{E U 272628}$ & MHNG INVE36298 \\
\hline Hydrozoa, Siphonophorae & Agalma elegans & EU272542 & AY937313 & YPM 35029 \\
\hline Hydrozoa, Siphonophorae & Apolemia sp. & EU272546 & AY937331 & YPM 35090 \\
\hline Hydrozoa, Siphonophorae & Cordagalma cordiforme & EU272555 & AY937317 & YPM 35032 \\
\hline Hydrozoa, Siphonophorae & Halistemma rubrum & EU272566 & AY937358 & YPM 35359 \\
\hline Hydrozoa, Siphonophorae & Nanomia bijuga & EU272576 & AY937338 & YPM 35043 \\
\hline Hydrozoa, Siphonophorae & Nectopyramis sp. & AY026377 & AF358068 & \\
\hline Hydrozoa, Siphonophorae & Physophora hydrostatica & EU272582 & AY937342 & YPM 35046 \\
\hline Hydrozoa, Siphonophorae & Sulculeolaria quadrivalvis & EU272594 & AY937353 & YPM 35357 \\
\hline Hydrozoa, Stylasteridae & Crypthelia cryptotrema & EU272558 & EU27264I & USNMI 027758 \\
\hline Hydrozoa, Stylasteridae & Lepidopora microstylus & EU272572 & EU272644 & USNMI027724 \\
\hline Hydrozoa, Stylasteridae & Pseudocrypthelia pachypoma & $\overline{\text { EU272589 }}$ & $\overline{\text { EU272643 }}$ & USNMI027728 \\
\hline Hydrozoa, Stylasteridae & Adelopora crassilabrum & EU27254I & EU272642 & USNMI027760 \\
\hline Hydrozoa, Trachylina & Limnocnida tanganyicae & AY920795 & $\overline{\text { AY920755 }}$ & \\
\hline Hydrozoa, Trachylina & Maeotias marginata & EU247810 & & \\
\hline Hydrozoa, Trachylina & Olindias phosphorica & EU247808 & AY920753 & MHNG INVE298I I \\
\hline Scyphozoa, Coronatae & Atolla vanhoeffeni & AY026368 & AFI00942 & \\
\hline Scyphozoa, Coronatae & Nausithoe rubra & AY920776 & AF358095 & \\
\hline Scyphozoa, Rhizostomea & Catostylus sp. & AY920777 & AF358100 & \\
\hline Scyphozoa, Semaeostomeae & Chrysaora melanaster & $\overline{\mathrm{AY} 920780}$ & $\overline{\mathrm{AF} 358099}$ & \\
\hline Scyphozoa, Semaeostomeae & Aurelia sp. & EU272547 & EU272604 & \\
\hline Scyphozoa, Semaeostomeae & Phacellophora camtschatica & AY920778 & AF358096 & \\
\hline Staurozoa, Stauromedusae & Craterolophus convolvulus & AY92078I & AY845344 & \\
\hline Staurozoa, Stauromedusae & Haliclystus octoradiatus & $\mathrm{AHOI} 4894$ & AY845346 & \\
\hline Staurozoa, Stauromedusae & Haliclystus sanjuanensis & AY920782 & $\overline{\mathrm{AF} 358102}$ & \\
\hline \multicolumn{5}{|l|}{ Myxozoa } \\
\hline Malacosporea & Buddenbrockia plumatellae & & A) 937883 & \\
\hline
\end{tabular}


Table I: Taxon and sequence list (Continued)

\begin{tabular}{|c|c|c|c|}
\hline Myxosporea & Henneguya salminicola & AY302726 & \\
\hline Myxosporea & Kudoa trifolia & AM490336 & AMI83300 \\
\hline Myxosporea & Kudoa unicapsula & AM490335 & AM490334 \\
\hline Myxosporea & Myxobolus cerebralis & & EF37048I \\
\hline Myxosporea & Myxobolus dogieli & & EU003978 \\
\hline Myxosporea & Parvicapsula limandae & & EF429096 \\
\hline \multicolumn{4}{|l|}{ Outgroups } \\
\hline \multicolumn{4}{|l|}{ Choanoflagellida } \\
\hline Codonosigidae & Monosiga brevicollis & AY026374 & AF084618 \\
\hline Salpingoecidae & Salpingoeca infusionum & $\underline{A Y 026380}$ & $\underline{A F I 00941}$ \\
\hline \multicolumn{4}{|l|}{ Ctenophora, } \\
\hline Cyclocoela & Beroe ovata & AY026369 & AF293694 \\
\hline Cyclocoela & Mnemiopsis leidyi & $\underline{A Y 026373}$ & $\underline{\text { AF293700 }}$ \\
\hline Typhlocoela & Pleurobrachia bachei & AY026378 & AF293677 \\
\hline \multicolumn{4}{|l|}{ Fungi } \\
\hline Ascomycota & Candida albicans & X70659 & $\times 53497$ \\
\hline Ascomycota & Saccharomyces cerevisiae & 101355 & $\underline{M 27607}$ \\
\hline Basidiomycota & Tricholoma matsutake & $\underline{\text { U62964 }}$ & $\underline{\text { U62538.I }}$ \\
\hline Mucoromycotina & Mucor racemosus & A) 271061 & A]271061 \\
\hline \multicolumn{4}{|l|}{ Porifera, } \\
\hline Calcarea & Leucosolenia sp. & AY026372 & AFI00945 \\
\hline Demospongia & Mycale fibrexilis & AY026376 & $\underline{\mathrm{AFI} 00946}$ \\
\hline Demospongia & Suberites ficus & AY02638I & AFI00947 \\
\hline
\end{tabular}

A complete list of sequences used in the analyses with GenBank accession numbers and museum voucher numbers. Bold numbers indicate new sequences generated for this study. KUMIP = University of Kansas Museum of Invertebrate Paleontology, KUNHM = University of Kansas Natural History Museum, MHNG = Muséum d'histoire naturelle de Genève, YPM = Yale Peabody Museum, USNM = US National Museum of Natural History.

zoans, is consistent with previously reported studies using the same marker [2-4], but raises similar concerns of longbranch attraction [5].

\section{Test of long-branch attraction}

Myxozoans and Polypodium have unusually high rates of evolution in their $18 \mathrm{~S}$ and $28 \mathrm{~S}$ rDNA sequences relative to the other sampled taxa. To investigate the influence of myxozoans on the placement of Polypodium, we removed the myxozoans from our three datasets and re-ran each analysis. Under the ML analysis of $18 \mathrm{~S}$ rDNA, the removal of myxozoans results in the placement of Polypodium nested within Cnidaria (Figure 3B and Additional file 3B). This result suggests that the placement of Polypodium at the base of bilaterians in the $18 \mathrm{~S}$ analysis (Figure 3A) was indeed an artifact of LBA. The placement of Polypodium within Cnidaria was not effected by the removal of myxozoans in the 28S (Additional file 6) and combined datasets (Additional file 7).

To investigate the possible role of LBA on myxozoan placement, we removed Polypodium from the combined ML analyses and found that it did not affect the position of Myxozoa at the base of the Bilateria (not shown). Given that bilaterians also form long branches, we tried removing all bilaterian sequences in the combined ML analysis. This resulted in a Myxozoa + Polypodium clade nested within Cnidaria (not shown). However, when Polypodium and bilaterians were removed, myxozoans fell outside the cnidarians (not shown). Similar effects of myxozoan placement to long-branches were also found in parsimony analyses of the combined dataset (not shown).

\section{Discussion}

\section{Polypodium is a cnidarian}

Our metazoan dataset of $18 \mathrm{~S}$ and partial $28 \mathrm{~S}$ rDNA sequences, with a large taxonomic sample of cnidarians, places Polypodium within a monophyletic Cnidaria. This accords with the fact that Polypodium possesses nematocysts $[17,18]$ and a cnidarian-like body plan $[7-9,12]$. The precise placement of Polypodium within Cnidaria is less certain. The ML combined analysis places Polypodium as sister to Hydrozoa (Figure 2A), a hypothesis consistent with the suggestion that Polypodium be considered a separate class of cnidarians, Polypodiozoa [1]. By contrast, the combined parsimony analysis (Figure $2 \mathrm{~B}$ ) and the $\mathrm{ML}$ analyses of $28 \mathrm{~S}$ alone (Additional file 2 and 6) place Polypodium within the hydrozoan clade Leptothecata. Given that leptothecates have relatively high rates of evolution within hydrozoans, one possible explanation for the conflicting hypotheses is that the placement of Polypodium within leptothecates is an artifact of LBA and that the combined data, in conjunction with the ML approach (Figure 2A), overcame this localized LBA artifact. 
A

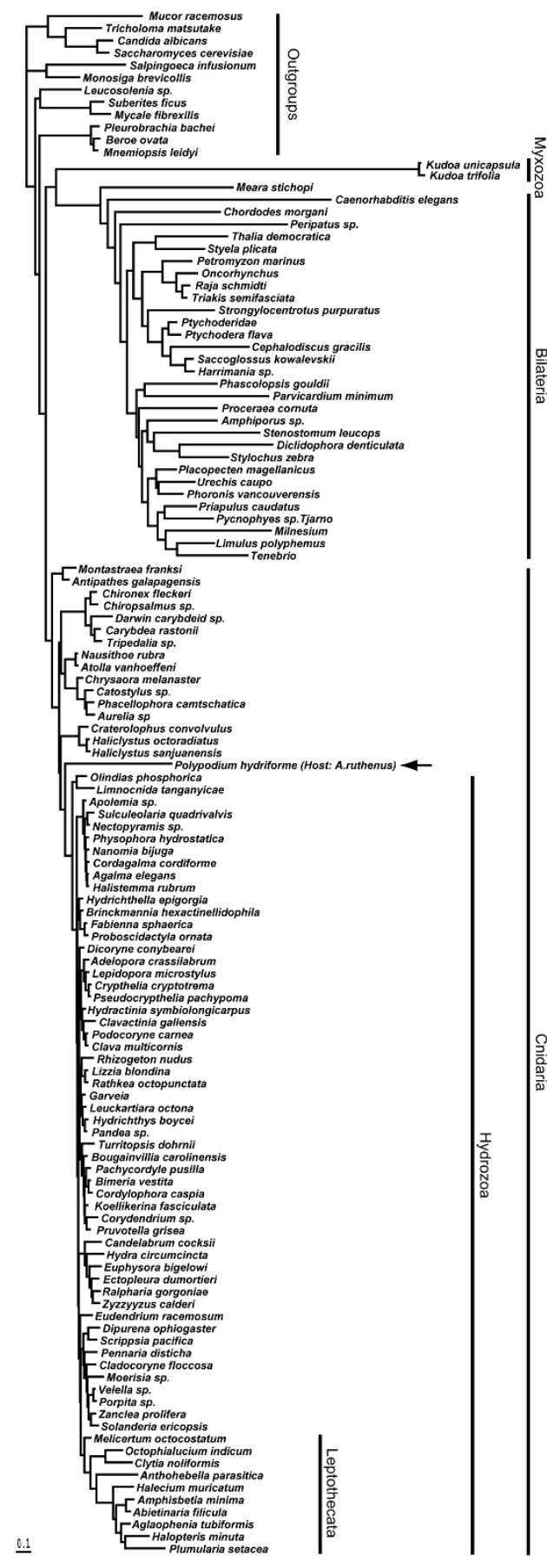

$\mathrm{B}$

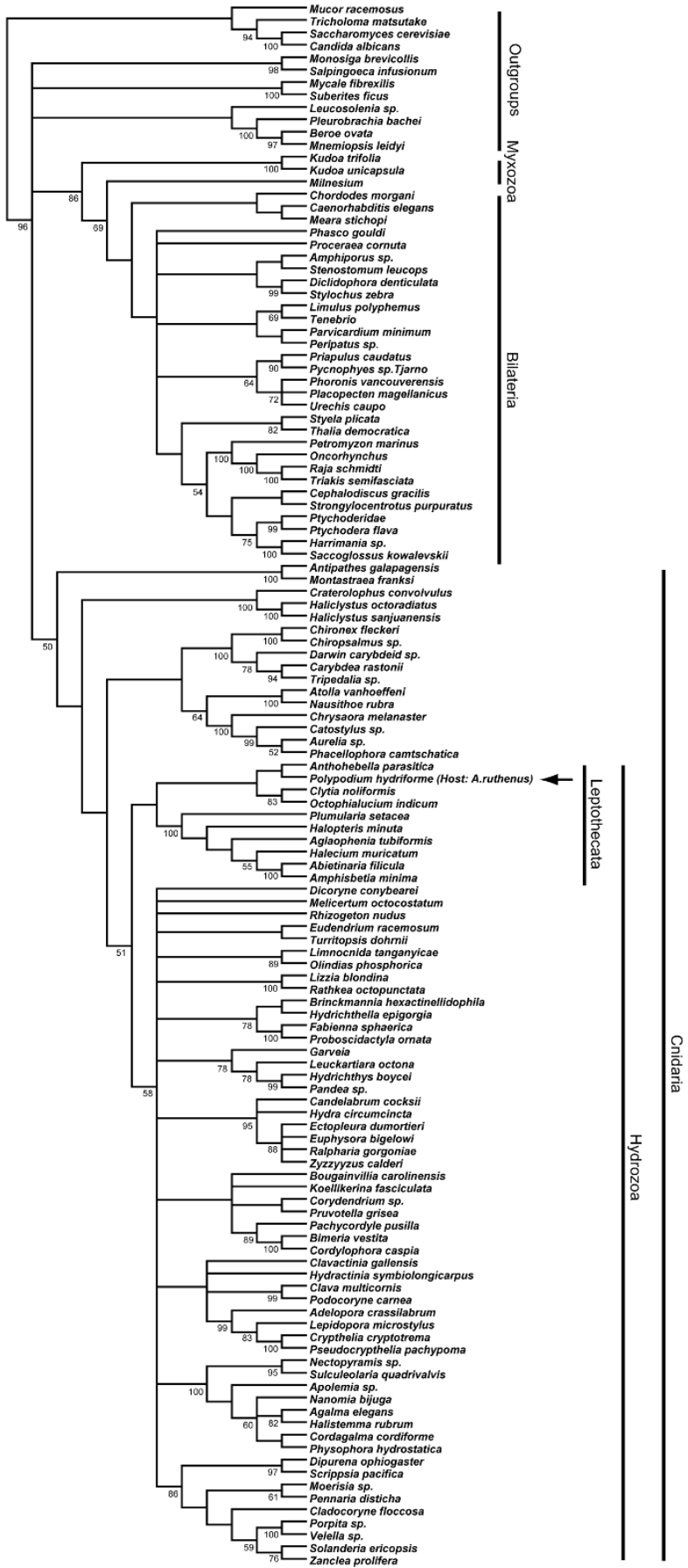

Figure 2

Phylogenetic hypotheses of relationships among I 26 metazoan taxa, based on a combined analysis of nearly complete 18S and partial 28S rDNA sequences. Arrow indicates Polypodium taxa. A) Maximum likelihood topology. The assumed model $(\mathrm{GTR}+\mathrm{I}+\mathrm{G})$ has six substitutions rates estimated from the data (A-C, I. I786; A-G, 3.3654; A-T, I.7283; C-G, 0.7403; C-T, 4.7803; G-T, I.0000), an assumed proportion of invariant sites (0.1692) and a gamma shaped parameter or (0.5584). The length of the bar indicates 0.1 substitutions per site. Bootstrap values for this topology are indicated on the cladogram in Additional file I. B) Strict consensus of 32 trees of length 25I4I from a parsimony analyses. Bootstrap values of 50 or greater are indicated. 
A

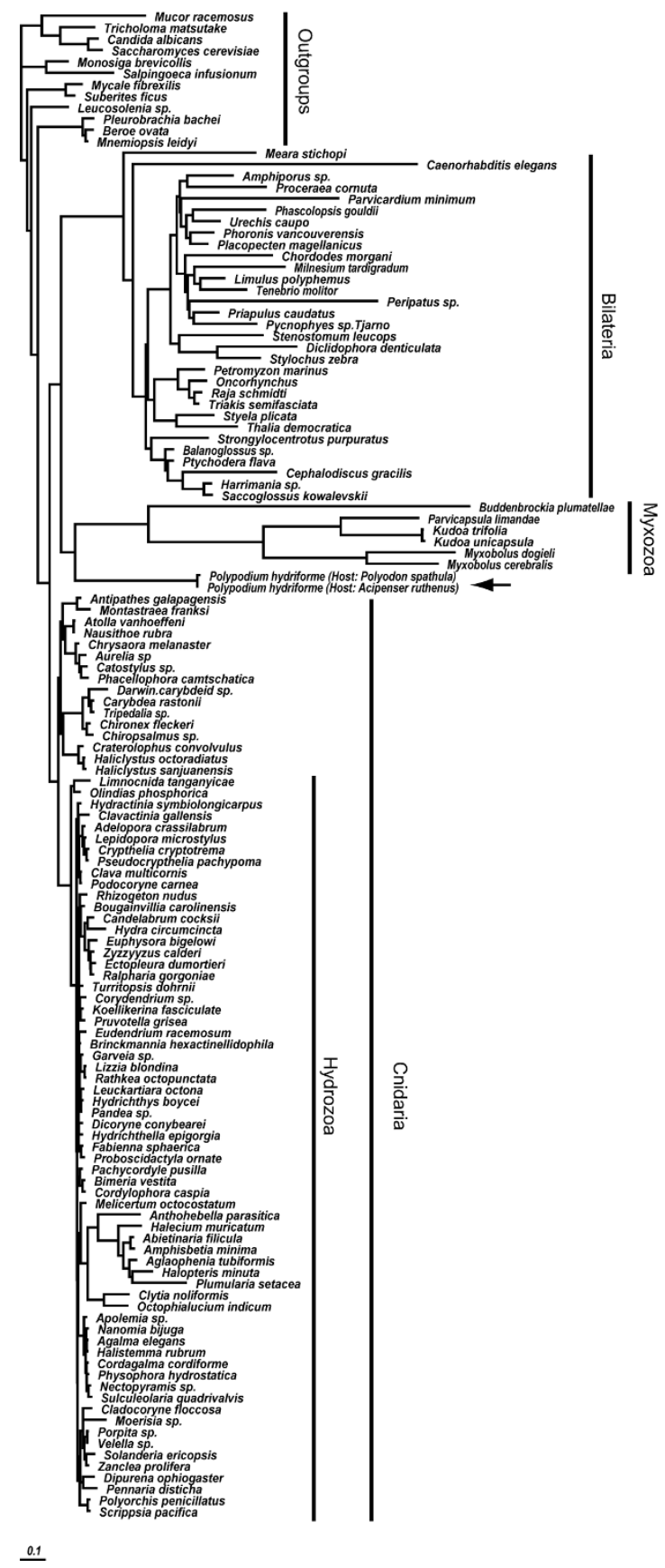

B

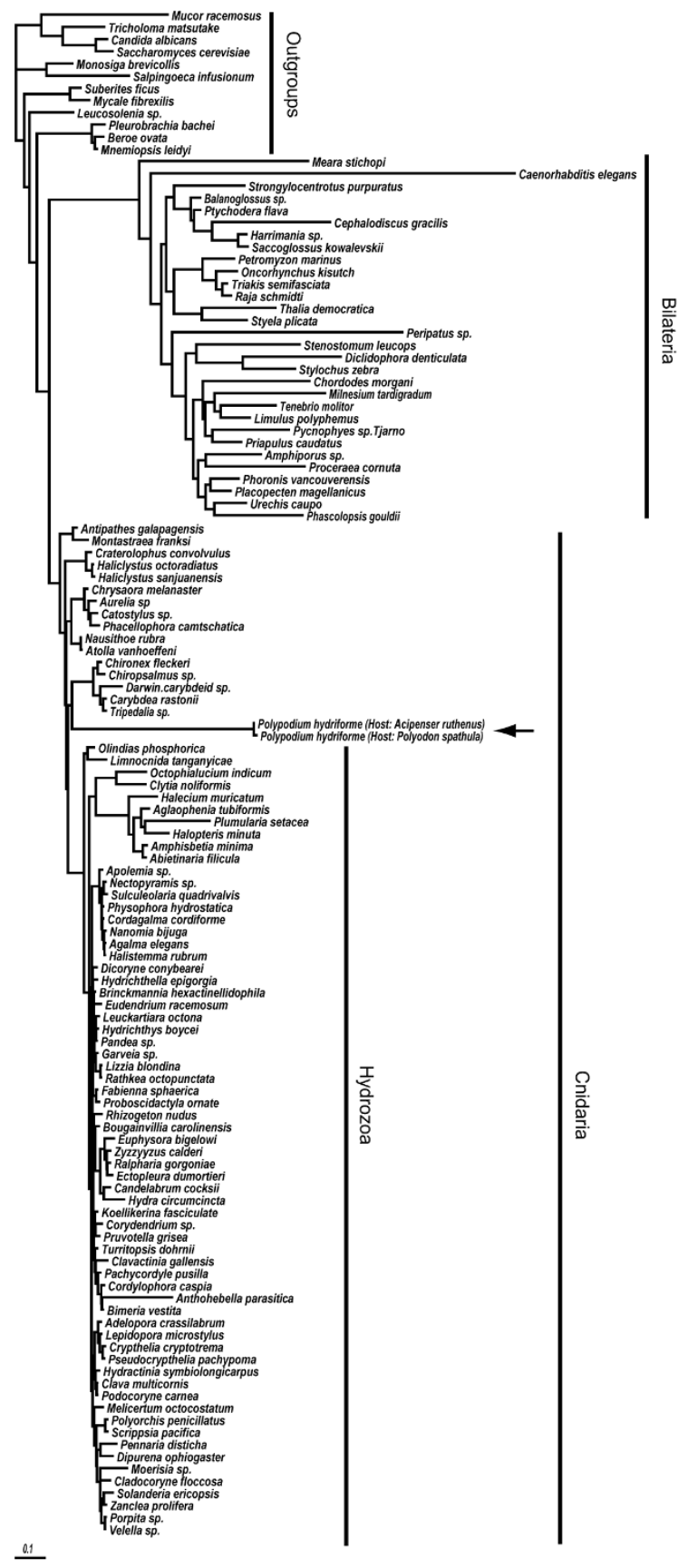

Figure 3

ML topologies of metazoan relationships of nearly complete I8S rDNA sequences. Arrow indicates Polypodium taxa. Bootstrap values for both topologies are indicated on the cladograms in Additional file 3. A) I32 taxa including 6 myxozoan taxa and two Polypodium taxa. The assumed model $(G T R+I+G)$ has six substitutions rates estimated from the data (A-C, I.407I; A-G, 3.3470; A-T, I.690 I; C-G, 0.84888; C-T, 4.7638; G-T, I.0000), an assumed proportion of invariant sites (0.1757) and a gamma shaped parameter or (0.5837). B) Same dataset as (A) but with the 6 myxozoan taxa removed. The assumed model $(G T R+I+G)$ has six substitutions rates estimated from the data (A-C, I.4III5; A-G, 3.3559; A-T, I.7502; C-G, 0.8342; C-T, 4.8554; G-T, I.0000), an assumed proportion of invariant sites (0.2464) and a gamma shaped parameter or $(0.6326)$. The length of the bar indicates 0.1 substitutions per site. 


\section{Evolution of Polypodium life-history characters}

Although the fresh water habitat of Polypodium is unusual for cnidarians, it is not unheard of, especially within hydrozoans. For instance, the model organism Hydra and the jellyfish Craspedacusta are both exclusively fresh-water hydrozoans. Hydra and Craspedacusta are distantly related [25] and our analyses do not indicate a close phylogenetic affinity of Polypodium to either of the clades containing these taxa. Thus, it appears that in the evolution of cnidarians, invasion to fresh-water habitats has happened at least three separate times.

Although Polypodium is the only known intracellular cnidarian parasite, other cnidarians have adopted parasitic life-styles [11,26-29]. For example, parasites belonging to the Narcomedusae (Hydrozoa) have been reported to live in the stomach cavities of other narcomedusae $[11,27]$ and anthomedusae [27]. In addition, the sea anemone Edwardsiella lineata parasitizes the stomach cavity of the ctenophore Mnemiopsis leidyi [28] and the anemone Peachia quinquecapitata is reported to parasitize the stomachs of hydromedusa [29].

\section{Effects of long-branch attraction}

The well-documented effects of long-branch attraction artifacts (reviewed in Bergsten [30]) are particularly concerning when investigating relationships amongst earlydiverging metazoans, where rates between lineages vary greatly [22]. Suggestions for avoiding LBA artifacts include choice of appropriate markers $[31,32]$, increased taxonomic sampling to effectively break up long branches $[33,34]$ and utilization of best-fit models that incorporate rate variation [21-23]. Previous conflicting reports that show Polypodium and myxozoans form a sister taxon to Bilateria [2-4] can be explained by limited taxon sampling and an inadequate number of informative characters in their analyses, both of which confound long-branch problems. In this study, the increased taxonomic sampling of cnidarians and the addition of $28 \mathrm{~S}$ rDNA sequence data proved critical to placing the highly divergent Polypodium taxon within Cnidaria. The choice of optimality criteria (ML vs. parsimony) both supported Polypodium as a cnidarian but did affect the placement within Cnidaria.

\section{Polypodium and Myxozoa}

Our analyses are inconclusive in the placement of Myxozoa within metazoans. We found that myxozoans consistently grouped with long-branched taxa and that removal of long-branches resulted in myxozoans being placed to the next longest branch. For example myxozoans group with Polypodium in the absence of Bilateria and group with Bilateria in the absence of Polypodium (not shown).

Jimenez-Guri et al. [24] sampled the myxozoan, Buddenbrockia, and found it to fall within Cnidaria, as the sister group to two hydrozoan representatives and a single scyphozoan. Previous studies have suggested a sister group relationship between cnidarians and myxozoans [2-4], and some morphological evidence has been used to support this view [35]. Although our present study does not support this relationship, further investigation is merited. Myxozoans are a highly diverse group (reviewed in Kent et al. [36]) that comprise two clades, the Myxosporea and the Malacosporea [37]. We were only able to include $28 \mathrm{~S}$ rDNA sequences from myxosporeans, although the malacosporean Buddenbrockia was included in our $18 \mathrm{~S}$ analysis and found to group with other myxozoans and outside of Cnidaria. Future studies with a comprehensive sampling of myxozoans together with Polypodium, in a dataset that includes a large taxonomic sampling of cnidarians, should shed further light on the relationships between myxozoans and Polypodium.

\section{Conclusion}

Although previous molecular phylogenetic hypotheses conflicted with the traditional interpretation of cnidarian affinity for Polypodium, the molecular evidence we present, using an augmented dataset, ultimately confirms and reconciles this traditional hypothesis and suggests that Polypodium is indeed a cnidarian. This study also reaffirms the importance to large taxonomic sampling and inclusion of additional informative characters for avoiding longbranch attraction artifacts.

\section{Methods}

DNA isolation, amplification and sequencing

Genomic DNA was extracted using Qiagen DNeasy kits according to manufacturer's protocol (QIAGEN Inc., Mississauga, ON) or a standard phenol/chloroform protocol. The latter method involved tissue digestion with proteinase $\mathrm{K}(20 \mathrm{mg} / \mathrm{ml})$ in a lysis buffer (20 mM Tris-CL pH 8.0, 5 mM EDTA pH 8.0, $400 \mathrm{mM} \mathrm{NaCl}, 2 \%$ SDS), extraction with phenol/chloroform (1:1), precipitation with $2.5 \mathrm{vol}$. $95 \% \mathrm{EtOH}$, and elution in TE or $\mathrm{H}_{2} \mathrm{O}$.

An approximately $1.8 \mathrm{~kb}$ portion of the gene coding for $18 \mathrm{~S}$ was amplified and sequenced with universal eukaryotic primers as described by Medlin et al. [38], with the annealing temperature modified to $57^{\circ} \mathrm{C}$. With the exception of Polypodium samples, a nearly complete, roughly 3 $\mathrm{kb}$ portion of the gene coding for $28 \mathrm{~S}$ was amplified and sequenced with an approach modified from that reported in Collins et al. [25]. $28 \mathrm{~S}$ was directly amplified in two fragments with combinations of primers F63mod+R2077sq and F1379+R3264 from Medina et al. [39] or newly developed medusozoan specific primers F97+R2084 and F1383+R3238 (F97: CCYYAGTAACGGCGAGT, R2084: AGAGCCAATCCTTTTCC, F1383: GGACGGTGGCCATGGAAGT, and R3238: SWACAGATGGTAGCTTCG). Amplifications of $28 \mathrm{~S}$ were conducted 
with the following thermal profile: 4 minutes at $94^{\circ} \mathrm{C} ; 30$ cycles of 30 seconds at $94^{\circ} \mathrm{C}, 1$ minute at $45^{\circ} \mathrm{C}$, and 3 minutes at $72^{\circ} \mathrm{C}$; and 10 minutes at $72^{\circ} \mathrm{C}$. For Polypodium, a portion of the $5^{\prime}$ end of $28 \mathrm{~S}$ (approx. 0.8-1.0 kbps) was amplified using two universal metazoan primers (fw1and rev2) as reported by Sonnenberg et al. [40]. Sequencing was carried out using amplification primers and F635sq and R635sq from Medina et al. [39].

All gene fragments were purified and sequenced by Cogenics, Inc. (Houston, TX) and assembled and edited using Sequencher v4.5 (Gene Code Co., 2005). Sequences for each marker were aligned using the program MUSCLE [41]. The $28 \mathrm{~S}$ sequence alignment was then trimmed to reflect only that region which included sequence data for Polypodium. This trimmed $28 \mathrm{~S}$ dataset was analyzed separately and used in conjunction with the complete $18 \mathrm{~S}$ sequences to create the combined dataset.

\section{Phylogenetic analyses}

Phylogenetic analyses were performed using both maximum likelihood (ML) and parsimony criteria. ML searches were performed using GARLI v0.951.OsX-GUI [42] under an assumed GTR model with rates estimated from the data. The assumed model of nucleotide substitution was selected by using the Akaike Information Criterion (AIC) as implemented in ModelTest [43]. Each run was repeated 10 times from random starting trees using default termination conditions. Each run gave identical topologies and similar likelihood scores. 100 bootstrap replications were run in GARLI v0.951.0sX-GUI [42] under the same parameters.

To assess the effect that omitting length-variable regions has on topology, we removed these regions from the combined dataset, using the less stringent settings of Gblocks [44]. This dataset contained 126 metazoan taxa, 2415 characters, 1391 of which are parsimony informative. We found that removal of length-variable regions had no effect on the placement of Polypodium and minimal effect on overall topology in our combined ML analyses (Additional file 8). Therefore we performed all other analyses with the complete datasets, including the more variable regions.

Parsimony analyses were performed using TNTv.1.1 [45]. Separate tree searches were performed with gaps coded as missing and gaps coded as a fifth state. However, with one exception (see results for myxozoan placement with $18 \mathrm{~S}$ data) there was no significant difference in topology. Numerous search methods available in TNT were utilized to search the tree space but the following approach was found to consistently recover trees with minimum lengths from our datasets. The implemented search was a driven new technology search with a random seed of 0 (where 0 = time). Default settings for sectorial searches (RSS and CSS) and tree fusing were used [46], with 5 replicates per repetition, and a requirement that the global optimum be found 20 times. TBR branch swapping was performed on the resulting trees and a strict consensus was calculated. TNT was used to calculate standard bootstrap values (1000 replicates). Alignments and trees for 18S, 28 S and combined datasets have been submitted to TreeBASE http://www.treebase.org/treebase/index.html.

\section{Authors' contributions}

This study was inspired by the work of EVR and originally conceived by EVR, AL and AGC. NME performed most of the data collection and submission of the new sequences. NME and PC performed the analyses. PC took the lead in organizing the study and drafting the manuscript with contributions from NME. All other authors provided comments and suggestions and approved the final manuscript.

\section{Additional material}

\section{Additional file 1}

$M L$ topology of relationships based on combined data. ML topology identical to Figure $1 \mathrm{~A}$ but as a cladogram showing bootstrap values.

Click here for file

[http://www.biomedcentral.com/content/supplementary/1471-

2148-8-139-S1.pdf]

\section{Additional file 2}

ML topology of relationships based on partial $28 S$ rDNA sequences. Relationships of 128 metazoan taxa were analyzed with partial 28S rDNA sequences.

Click here for file

[http://www.biomedcentral.com/content/supplementary/14712148-8-139-S2.pdf]

\section{Additional file 3}

$M L$ topologies of relationships based on $18 S$ data with and without myxozoans. ML topologies identical to Figure 3 but as cladograms showing bootstrap values.

Click here for file

[http://www.biomedcentral.com/content/supplementary/14712148-8-139-S3.pdf]

\section{Additional file 4}

Parsimony topology of relationships based on $18 S \mathrm{rDNA}$ sequences. This parsimony analysis of $18 \mathrm{~S} r \mathrm{DNA}$ sequences included 132 taxa with gaps coded as a fifth state.

Click here for file

[http://www.biomedcentral.com/content/supplementary/14712148-8-139-S4.pdf] 


\author{
Additional file 5 \\ Parsimony topology of relationships based on $18 S \mathrm{rDNA}$ sequences. This \\ parsimony analysis of $18 \mathrm{~S} r \mathrm{DNA}$ sequences included 132 taxa with gaps \\ coded as missing. \\ Click here for file \\ [http://www.biomedcentral.com/content/supplementary/1471- \\ 2148-8-139-S5.pdf]

\section{Additional file 6} \\ ML topology of relationships excluding myxozoans, based on partial $28 \mathrm{~S}$ \\ $r D N A$ data. This ML analysis of partial 28S rDNA sequences excluded \\ myxozoan taxa. \\ Click here for file \\ [http://www.biomedcentral.com/content/supplementary/1471- \\ 2148-8-139-S6.pdf]

\section{Additional file 7} \\ $M L$ topology of relationships excluding myxozoans, based on combined \\ data. This ML analysis of partial $28 \mathrm{~S} r \mathrm{DNA}$ and $18 \mathrm{~S}$ sequences excluded \\ myxozoan taxa. \\ Click here for file \\ [http://www.biomedcentral.com/content/supplementary/1471- \\ 2148-8-139-S7.pdf]

\section{Additional file 8} \\ $M L$ topology of relationships based on combined data. This analysis of 126 \\ metazoan taxa was based on combined $18 S$ and partial $28 S$ rDNA with \\ length variable sequences removed. \\ Click here for file \\ [http://www.biomedcentral.com/content/supplementary/1471- \\ 2148-8-139-S8.pdf]
}

\section{Acknowledgements}

We thank S. Ash Bullard, Jan Dean, Bobby Reed and Ron Bruch for contributions of Polypodium specimens. We also thank Peter Schuchert, Casey Dunn and Michael Dawson for contributions of other cnidarian specimens. NME acknowledges the instructors at the 2007 Ohio State Cladistics workshop, especially P. Goloboff, for help with TNT. We thank M. Holder for discussions, B. Bentlage, B. Lieberman, A. Nawrocki, and five anonymous reviewers for helpful comments on earlier versions of this manuscript. This work was supported by grants from NSF AToL EF-0531779 (to PC and AGC) and support for AL from NSF (PEET DEB-9978086) and FAPESP (06/ 02960-8/0582I-9/60327-0).

\section{References}

I. Raikova EV: On the systematic position of Polypodium hydriforme Ussov, (Coelenterata). In Porifera and Cnidaria Contemporary state and perspectives of investigations Edited by: Koltum VM, Stepanjants SD. Leningrad: Zoological Institute of Academy of Sciences of USSR; 1988: I 16-122.

2. Siddall ME, Martin DS, Bridge D, Cone DM, Desser SS: The demise of a phylum of protests: Phylogeny of Myxozoa and other parasitic Cnidaria. J Parasitol 1995, 81 1:961-967.

3. Siddall ME, Whiting MF: Long-branch abstractions. Cladistics 1999, 15:9-24.

4. Zrzavý J, Hypša V: Myxozoa, Polypodium, and the origin of the Bilateria: The phylogenetic position of "Endocnidozoa" in the light of the rediscovery of Buddenbrockia. Cladistics 2003, 19(3):164-169.

5. Hanelt B, Van Schyndel D, Adema CM, Lewis L, Loker ES: The phylogenetic position of Rhopalura ophiocomae (Orthonectida) based on $18 \mathrm{~S}$ ribosomal DNA sequence analysis. Mol Biol Evol 1996, 13: II87-II9I.

6. Raikova EV: Life cycle and systematic position of Polypodium hydriforme Ussov (Coelenterata), a cnidarian parasite of the eggs of Acipenseridae. Publ Seto Mar Biol Lab 1973, 20:165-173.

7. Raikova EV: Morphology, ultrastructure and development of the parasitic larva and its surrounding trophamnion of Polypodium hydriforme Ussov (Coelenterata). Cell Tissue Res 1980, 206(3):487-500.

8. Raikova EV: Life cycle, cytology, and morphology of Polypodium hydriforme, a coelenterate parasite of the eggs of acipenseriform fishes. J Parasitol I994, 80(I): I-22.

9. Raikova EV, Suppes VC, Hoffmann GL: The parasitic coelenterate, Polypodium hydriforme Ussov, from the eggs of the American acipenseriform Polyodon spathula. J Parasitol 1979, 65(5):804-810.

10. Berrill NJ: Development and medusa-bud formation in the Hydromedusae. Quart Rev Biol 1950, 25:292-316.

II. Bouillon J: Considérations sur les dévéloppement des narcoméduses et sur leur position phylogenétique. Indo-Malayan Zool 1987, 4:189-278.

12. Hyman L: The invertebrates. I. Protozoa through Ctenophora. New York and London: McGraw-Hill. 1940

13. Schimkevitch VM: Essay on contemporary state of the problem of the development of Hydrozoa. Vestnik Estestvoznanija 1890, 4:17I-176.

14. Lipin A: Geschlechtliche Form, Phylogenie und systematische Stellung von Polypodium hydriforme Ussov. Zool Jahrb Anat 1925, 47:54I-635.

15. Bouillon J, Medel MD, Pagès F, Gili JM, Boero F, Gravili C: Fauna of the Mediterranean Hydrozoa. Scientia Marina 2004, 68(Suppl 2):5-438.

16. Bouillon J, Gravili c, Pagès F, Gili JM, Boero F: An introduction to Hydrozoa. Paris: Publications Scientifiques du Muséum, Paris; 2006.

17. Raikova EV: Fine structure of the nematocytes of Polypodium hydriforme Ussov (Cnidaria). Zoologica Scripta 1990, I9(I): I-I I.

18. Ibragimov A, Raikova E: Nematocysts of Polypodium hydriforme, a cnidarian parasite of acipenseriform fishes. Hydrobiologia 2004, 531:165-171.

19. Dellaporta SL, Xu A, Sagasser S, Jakob W, Moreno MA, Buss LW, Schierwater B: Mitochondrial genome of Trichoplax adhaerens supports Placozoa as the basal lower metazoan phylum. Proc Natl Acad Sci USA 2006, 103(23):875I-8756.

20. Collins AG: Phylogeny of Medusozoa and the evolution of cnidarian life cycles. J Evol Biol 2002, I5(3):4I 8-432.

21. Huelsenbeck JP: Is the Felsenstein zone a fly trap? Syst Biol 1997, 46:247-264.

22. $\mathrm{Kim} \mathrm{JH}, \mathrm{Kim} \mathrm{W}$, Cunningham CW: A new perspective on lower metazoan relationships from I8S rDNA sequences. Mol Biol Evol 1999, 16:423-427.

23. Cunningham $\mathrm{CW}$, Zhu H, Hillis DM: Best-fit maximum likelihood models for phylogenetic inference: Empirical tests with known phylogenies. Evolution 1998, 52:978-987.

24. Jimenez-Guri E, Philippe H, Okamura B, Holland PWH: Buddenbrockia is a cnidarian worm. Science 2007, $317(5834): 116-1 \mid 18$.

25. Collins AG, Schuchert P, Marques AC, Jankowski T, Medina M, Schierwater B: Medusozoan phylogeny and character evolution clarified by new large and small subunit rDNA data and an assessment of the utility of phylogenetic mixture models. Syst Biol 2006, 55(1):97-II5.

26. Osborn DA: Cnidarian "parasites" on Solmissus incisa, a Narcomedusa. Sci Mar 2000, 64:157-163.

27. Pagès F, Corbera J, Lindsay D: Piggybacking pycongonids and parasitic narcomedusae on Pandea rubra (Anthomedusae, Pandeidae). Plankton Benthos Res 2007, 2(2):83-90.

28. Bumann D, Puls G: Infestation with larvae of the sea anemone Edwardsia lineata affects nutrition and growth of the ctenophore Mnemiopsis leidyi. Parasitology 1996, I I3: I23-128.

29. Spaulding JG: The life cycle of Peachia quinquecapitata, an anemone parasitic on medusae during its larval development. Biol Bull 1972, I 43(2):440-453.

30. Bergsten J: A review of long-branch attraction. Cladistics 2005, 21:163-193.

31. Rokas A, King N, Finnerty J, Carroll SB: Conflicting phylogenetic signals at the base of the metazoan tree. Evol Dev 2003, 5(4):346-359. 
32. Xiang QY, Moody ML, Soltis DE, Fan C, Soltis PS: Relationships within Cornales and circumscription of Cornaceae - matK and rbcL sequence data and effects of outgroups and long branches. Mol Phyl Evol 2002, 24:35-57.

33. Hillis DM: Taxonomic sampling, phylogenetic accuracy, and investigator bias. Syst Biol 1998, 47:3-8.

34. Zwickl DJ, Hillis DM: Increased taxon sampling greatly reduces phylogenetic error. Syst Biol 2002, 5 I:588-598.

35. Raikova EV: Cytomorphological characters of Polypodium hydriforme and problems of myxozoan and cnidarian phylogeny. Tsitologiia 2005, 47(10):933-939.

36. Kent ML, Andree KB, Bartholomew JL, El-Matbouli M, Desser SS, Devlin RH, Feist SW, Hedrick RP, Hoffmann RW, Khattra J, Hallett SL, Lester RJG, Longshaw M, Oswaldo P, Siddall ME, Xiao C: Recent advances in our knowledge of the Myxozoa. J Eukaryot Microbiol 200I, 48(4):395-4I3.

37. Canning EU, Curry A, Feist SW, Longshaw M, Okamura B: A new class and order of myxozoans to accommodate parasites of bryozoans with ultrastructural observations on Tetracapsula bryosalmonae (PKX Organism). J Eukaryot Microbiol 2000, 47(5):456-468.

38. Medlin LH, Elwood HJ, Stickel S, Sogin ML: The characterization of enzymatically amplified eukaryotic I6S-like rRNA-coding regions. Gene 1988, 71:491-499.

39. Medina M, Collins AG, Silberman JD, Sogin ML: Evaluating hypotheses of basal animal phylogeny using complete sequences of large and small subunit rRNA. Proc Natl Acad Sci USA 200I, 98(1 8):9707-97|2.

40. Sonnenberg R, Nolte A, Tautz D: An evaluation of LSU rDNA DI-D2 sequences for their use in species identification. Front Zool 2007, 4(I):6.

4l. Edgar RC: MUSCLE: multiple sequence alignment with high accuracy and high throughput. Nucleic Acids Res 2004 32(5): I792-I797.

42. Zwickl DJ: Genetic algorithm approaches for the phylogenetic analysis of large biological sequence datasets under the maximum likelihood criterion. Austin: The University of Texas; 2006.

43. Posada D, Crandall KA: Modeltest: testing the model of DNA substitution. Bioinformatics 2000, 14:817-818.

44. Castresana J: Selection of conserved blocks from multiple alignments for their use in phylogenetic analysis. Mol Biol Evol 2000, I 7:540-552

45. Goloboff P, Farris J, Nixon K: TNT: Tree Analysis Using New Technology. I.Ith edition. Tucuman, Argentina: Published by the authors; 2003.

46. Goloboff P: Analyzing large data sets in reasonable times: Solutions for composite optima. Cladistics 1999, 14:415-428.
Publish with Bio Med Central and every scientist can read your work free of charge

"BioMed Central will be the most significant development for disseminating the results of biomedical research in our lifetime. "

Sir Paul Nurse, Cancer Research UK

Your research papers will be:

- available free of charge to the entire biomedical community

- peer reviewed and published immediately upon acceptance

- cited in PubMed and archived on PubMed Central

- yours - you keep the copyright
BioMedcentral 